\title{
Sylow Prime Group
}

\author{
T. Srinivasarao \\ Asst. Professor \\ Dept. of Math \\ University College of Science \& Technology \\ Adikavi Nannaya University
}

\author{
Mr. K. Revathi \\ Asst. Professor \\ Dept. of Math \\ University College of Science \& Technology \\ Adikavi Nannaya University
}

\begin{abstract}
Sylow $p$ - subgroup is a vital part of the discussion in any algebraic activity dealing with group theory. So, it is natural that, is every group can have Sylow $\boldsymbol{p}$ - subgroups or any specific distinction can be put forward that confirms a particular group is either a group having Sylow $p$ - subgroup or there is no single Sylow $p$ - subgroup. With a view to characterize the groups whether possessing a Sylow $p$ - subgroup or not here is an activity that progresses the discussion by one step ahead. This discussion leads to groups of order $p$ and order $p q$ that has applications in Galvan theory.
\end{abstract}

\section{INTRODUCTION:}

Suppose $n$ is a positive integer. By the fundamental theorem of arithmetic, either $n$ is a group of prime order or it is a product of primes expressible in a unique manner as $n=p_{1}{ }^{\alpha_{1}} p_{2}{ }^{\alpha_{2}} \ldots p_{k}{ }^{\alpha_{k}}$ where $p_{i}{ }^{\prime} s$ are prime numbers with the respective multiplicities $\alpha_{i}, 1 \leq i \leq k$.

Since each $p_{i}$ is a prime number, $1 \leq i \leq k$, there exists a cyclic group $G_{i}, 1 \leq i \leq k$ such that $\left|G_{i}\right|=p_{i}^{\alpha_{i}}, 1 \leq i \leq k$

Now, $G=G_{1} \times G_{2} \times \ldots \times G_{k}$

\section{Sylow Prime Group:}

Definition : if $G$ is a finite group, $p$ is a prime number such that $p^{n}|| G \mid$ and $p^{n+1}|| G \mid$, then any subgroup of $G$ of order $p^{n}$ is called a Sylow $p$ - subgroup of $G$.

Definition 2: a group $(G, *)$ is said to be a Sylow prime group if every non trivial subgroup of $G$ is a Sylow $p_{i}$ - subgroup for some prime factor $p_{i}$ of the order of $G$.

Since $|G|=n=p_{1}^{\alpha_{1}} p_{2}^{\alpha_{2}} \ldots p_{k}^{\alpha_{k}}$, and in view of Lagrange's theorem of finite groups, and properties of divisibility, it follows that $p_{i}^{\alpha_{i}}|| G \mid$ for each $1 \leq i \leq k$ and $p_{i+1}^{\alpha_{i+1}}|| G \mid$ by the unique representation of the integer $n$.

It is not necessary that there is a subgroup of $G$ of order $p_{i}{ }^{\alpha_{i}}$ for every $1 \leq i \leq k$. This confirms that every group of finite order is not a Sylow prime group.

To verify these observations, the following instances will show a finite group that admits the definition of Sylow prime group and another instance for not.

\section{Working on Sylow Prime Groups:}

Consider the symmetric group of order 6 or the symmetric group on 3 symbols.

$S_{3}=\left\{f_{1}, f_{2}, f_{3}, f_{4}, f_{5}, f_{6}\right\}$ where $f_{i}: A \rightarrow A$ is a bijection for each $1 \leq i \leq 6$ and $A=\{a, b, c\}$

$f_{1}=\{(a, a),(b, b),(c, c)\}$

$f_{2}=\{(a, b),(b, a),(c, c)\}$

$f_{3}=\{(a, a),(b, c),(c, b)\}$

$f_{4}=\{(a, c),(b, b),(c, a)\}$

$f_{5}=\{(a, b),(b, c),(c, a)\}$

$f_{6}=\{(a, c),(b, a),(c, b)\}$ 
The composition of mappings ' $\circ$ ' is the operation that makes $S_{3}$ a group such that $\left|S_{3}\right|=6=2^{1} \times 3^{1}$, the unique representation by fundamental theorem of arithmetic

It can be easily seen that $H_{1}=\left\{f_{1}, f_{4}\right\}, H_{2}=\left\{f_{1}, f_{5}, f_{6}\right\}$ are the only non trivial subgroups such that $\left|H_{1}\right|=2^{1}, 2^{2} \chi\left|S_{3}\right|$ and so, $H_{1}$ is a Sylow 2- subgroup of $S_{3}$

Similarly, $\left|H_{2}\right|=3^{1}, 3^{2} \gamma\left|S_{3}\right|$ and so, $H_{2}$ is a Sylow $3-$ subgroup of $S_{3}$

Take another instance.

$\square / 12=\{[1],[5],[7],[11]\}$ is a group under multiplication modulo 12 denoted by $\times_{12}$.

$|\square / 12|=4=2^{2}$

$H_{1}=\{[1],[11]\}$ is a non trivial subgroup of order $2^{1}$.

Also, $2^{2}|\square / 12|$ which shows $H_{1}$ is not a Sylow 2 - subgroup of $\square / 12$

So, this is an example of a group that is not a Sylow prime group.

The working (2.1) and (2.2) will confirm that all finite groups are not Sylow Prime Groups.

\section{REFERENCES}

[1] R.Gow, Sylow's proof of Sylow's theorem, Irish Math.Sco.Bull. (1994), 55 - 63

[2] L.Sylow, Theorems sur les groups de substitutions, Mathematische Annalen 5(1872), 584 - 594

[3] W. C. Waterhouse, The early proofs of Sylow's theorems, Arch.Hist.Exact Sci, 21(1979/80), 279-290

[4] William Fulton and Joe Harris. Representation Theory: A First Course. GTM 129, Springer, 1991 